\title{
Validation of a palliative care outcome measurement tool supplemented by neurological symptoms (HOPE + ): Identification of palliative concerns of neurological patients
}

Palliative Medicine 2019, Vol. 33(9) 1221-1231 (C) The Author(s) 2019 Article reuse guidelines: sagepub.com/journals-permission DOI: $10.1177 / 0269216319861927$ journals.sagepub.com/home/pmj

@SAGE

\author{
Kim Dillen ${ }^{1}$ D, Markus Ebke ${ }^{2,3}$, Andreas Koch ${ }^{1}$, Ingrid Becker ${ }^{4}$, \\ Christoph Ostgathe ${ }^{5}$, Raymond Voltz ${ }^{1,6,7,8}$ and Heidrun Golla ${ }^{1,6}$
}

\begin{abstract}
Background: There is growing interest to integrate palliative care and its structures into the care of neurological patients. However, in Germany there is no comprehensive assessment tool capturing the symptoms of patients with advanced neurological diseases.

Aim: To validate a newly developed palliative care measurement tool based on an extension of the validated core documentation system Hospice and Palliative Care Evaluation considering additional neurological issues (HOPE + ).

Design: Prospective, observational study using HOPE + and as external criteria, the Eastern Cooperative Oncology Group (ECOG) performance status and the 12 months "surprise" question (12-SQ) in a neurological population, and assessment for its construct validity and diagnostic accuracy.

Setting/participants: All newly admitted patients to the Department of Neurorehabilitation, Dr. Becker Rhein-Sieg-Clinic aged 18100 years (\#DRKS00010947).

Results: Data from 263 patients ( $63 \pm 14$ years of age) were analyzed. HOPE + revealed a moderately correlated six-factor structure $(r=-0.543-0.525)$. Correlation analysis to evaluate discriminant validity using ECOG as external criterion was high $(r s(261)=0.724$, $p<0.001$ ) and confirmed for severely affected patients by adding the 12-SQ ("No"-group: $48.00 \pm 14.92$ vs "Yes"-group: 18.67 \pm 7.57 , $p<0.009$ ). Operating characteristics show satisfactory diagnostic accuracy (area under the curve: $0.746 \pm 0.049,95 \%$ confidence interval $=0.650-0.842$ ).

Conclusion: HOPE + demonstrates promising psychometric properties. It helps to assess palliative care issues of patients in neurological settings and, in combination with the $12-\mathrm{SQ}$, conceivably conditions when to initiate the palliative care approach in a population underrepresented in palliative care structures so far.
\end{abstract}

\section{Keywords}

Palliative care concerns, neurological patients, symptom burden, prospective observational design, validation, outcome measurement

\footnotetext{
${ }^{1}$ Department of Palliative Medicine, Faculty of Medicine and University Hospital of Cologne, University of Cologne, Cologne, Germany ${ }^{2}$ Neurological Centre for Rehabilitation-MEDIAN-Clinics, Bad Salzuflen, Germany

${ }^{3}$ Dr. Becker Rhein Sieg Clinic, Nümbrecht, Germany

${ }^{4}$ Institute of Medical Statistics and Computational Biology (IMSB), Faculty of Medicine, University of Cologne, Cologne, Germany ${ }^{5}$ Department of Palliative Medicine, Universitätsklinikum Erlangen, Friedrich-Alexander-Universität Erlangen-Nürnberg (FAU), Erlangen, Germany
}

\footnotetext{
${ }^{6}$ Center for Integrated Oncology, Faculty of Medicine and University Hospital of Cologne, University of Cologne, Cologne, Germany ${ }^{7}$ Center for Clinical Trials, University of Cologne, Cologne, Germany ${ }^{8}$ Center for Health Services Research (ZVFK), Faculty of Medicine and University Hospital of Cologne, University of Cologne, Cologne, Germany

Corresponding author:

Kim Dillen, Department of Palliative Medicine, Faculty of Medicine and University Hospital of Cologne, University of Cologne, Kerpener Strasse 62, 50924 Cologne, Germany.

Email: kim.dillen@uk-koeln.de
} 


\section{What is already known about the topic?}

- Outcome measurements in palliative care have primarily been developed on the basis of the leading population in palliative care structures, that is, oncological patients.

- Detecting neurological patients in need of palliative care and its services remains challenging in Germany and many other countries due to the lack of a comprehensive measurement tool for this patient population.

\section{What this paper adds?}

- This validation study demonstrates promising psychometric properties of a new measurement tool $(\mathrm{HOPE}+)$ capturing palliative care concerns of neurological patients.

- $\mathrm{HOPE}+$ has potential for an improved prognosis estimation in neurological patients.

\section{Implications for practice, theory, or policy}

- $\mathrm{HOPE}+$ is an easy to handle outcome measurement tool feasible during clinical routine of neurologists.

- $\mathrm{HOPE}+$ helps to identify neurological patients in need of palliative care and its services.

\section{Introduction}

In recent years, there has been a gradual shift toward integrating patients other than cancer patients into palliative care, ${ }^{1,2}$ that is, the number of cancer patients cared for in German palliative and hospice care structures decreased from $95 \%$ in 2005 to $76 \%$ in 2017 while the number of patients diagnosed with incurable neurological diseases increased from $0.8 \%$ to $4.8 \%$. Yet, neurological patients are still underrepresented in German palliative and hospice care structures considering the high incidence and prevalence of severe neurological diseases including neurodegenerative or vascular diseases. This is also evident from the comparably low number of neurological patients in palliative care worldwide. For example, in the United States, only $8 \%$ of patients in palliative care structures have a neurological disease. ${ }^{3}$

Concurrently, on an international level, there is growing interest among neurologists to become more knowledgeable in palliative care and numerous studies advocate palliative care training for neurologists, ${ }^{1,4-8}$ and vice versa, the need for neurological expertise within palliative care health professionals. ${ }^{1,2,6}$ Pursuing this, a combined neuropalliative care approach is self-evident. An important prerequisite for integrating neurological patients into palliative care is a proper assessment tool, which recognizes palliative care concerns comprehensively. This will allow for the development of an effective palliative care treatment plan including timely advanced care planning, conversation on prognosis and disease course, and referral to palliative and hospice care structures. However, due to the different disease trajectories and health care needs of neurological patients compared to those of cancer patients, ${ }^{1,6}$ typical international palliative care assessment tools (e.g. Palliative Care Outcome Scale (POS), ${ }^{9}$ Edmonton Symptom Assessment Schedule, ${ }^{10}$ the Palliative Care Assessment) ${ }^{11}$ fail to capture the comprehensive symptom burden of neurological patients in need of palliative care. One reason is that palliative care outcome measurements were initially developed on a population mainly consisting of palliative cancer patients as they represented the main palliative care population during the modern palliative and hospice care movement. ${ }^{9-12}$ As such, they do not sufficiently evaluate neuropsychiatric symptoms, for example, cognitive deficits or behavioral changes. $6,9-11,13$ One example of such an outcome measurement, widely used in Germany, is the symptom checklist of the core documentation system Hospice and Palliative Care Evaluation (HOPE-SP-CL), which also includes an assessment of the Eastern Cooperative Oncology Group (ECOG) performance status. ${ }^{14-16}$ Previous research suggested that the HOPE-SP-CL might be appropriate for use in non-oncological patients such as neurological patients if extended by proper symptoms. ${ }^{14}$

In a first attempt to assess neuropsychiatric symptoms in palliative care patients with Glioblastoma, a previous study included free-text entries into the standard assessment (HOPE including HOPE-SP-CL and ECOG as well as the POS) $)^{9,14-19}$ and concluded that these assessment tools should be revised accordingly for such patient groups. ${ }^{20}$ The current study uses the neuropsychiatric categories found in this study ${ }^{20}$ and extends them with palliative care concerns of neurological patients described in previous studies. ${ }^{21-28}$ This resulted in a symptom checklist of 13 neurological items (which we will refer to as "HOPENeuro" for the remainder of the paper). The HOPE-Neuro in conjunction with the HOPE-SP-CL and ECOG (which we will name "HOPE+") serves as groundwork for development of an assessment tool suitable for neurological patients who might be in need of palliative care. One further aspect of this comprehensive assessment tool is the additional use of the 12 months "surprise" question (12SQ) as recommended by the German national S3 palliative guideline for oncological patients ${ }^{29}$ to predict an opportune time to initiate palliative care..$^{30} \mathrm{~A}$ poor prognosis estimation, that is, "No, I would not be surprised if 
my patient would die within the next 12 months" indicates instant integration of the palliative care approach into the care of the respective patient.

To explore the suitability of the HOPE + in combination with ECOG and 12-SQ, we here investigated its validity on a sample of neurological patients. We thus evaluated the diagnostic and psychometric properties of HOPE + and compared them to those of the well-established and validated HOPE-SP-CL. ${ }^{14}$

\section{Methods}

\section{Participants}

Patients between the ages of 18 and 100 (of all genders) were recruited from the Rehabilitation Center Dr. Becker Rhein-Sieg-Klinik, Department of Neurorehabilitation, in Nümbrecht over a 7-month period commencing in August 2016. All newly admitted patients were assessed by the attending neurologist (totaling M.E. as head of the department, one senior physician, and three assistant physicians) with 12-SQ and HOPE + including a 1-year follow-up to ascertain the patients' survival status. Only patients with "palliative care-relevant illnesses" were included into the analysis of the current study.

All study participants, or a legal representative, provided written informed consent. The study was approved by institutional local ethics review boards of the North Rhine Medical Chamber and the University Hospital of Cologne (\#16-118 on 10 August 2016), registered at the German Clinical Trials Register (\#DRKS00010947 on 10 August 2016) and in accordance with the Declaration of Helsinki. ${ }^{31}$

\section{Outcome measurements}

In Germany, palliative health care specialists routinely use the standardized documentation tool HOPE, developed in 1996 by a multi-disciplinary working group with annual revisions and extensions during a 3-month evaluation period since 1999.15,16,32 The core documentation tool assesses demographic data, diagnosis, course of treatment and therapeutic intervention, performance status, medication, and symptom burden. The latter has been evaluated categorically in 2001 using free-text entries for physical, psychological, nursing, social, and other problems resulting in the currently used 17-item HOPE-SP-CL for use by palliative health care specialists. ${ }^{15}$ In its current form, it covers physical symptoms (pain, nausea, vomiting, dyspnea, constipation, weakness, loss of appetite, tiredness), nursing problems (wound care, assistance with activities of daily living), psychological symptoms (feeling depressed, anxiety, tension, disorientation/confusion), and social needs (organization of care, overburdening of family), and concludes with an open-ended question for additional symptoms. The German version has been validated and evaluated using data from palliative care specialists in palliative care units, hospices, nursing services, oncology, and geriatric wards. ${ }^{14,33}$

In the current study, HOPE-Neuro was added to HOPESP-CL for use in neurological health care structures by health care professionals other than palliative care. ${ }^{13,20}$ HOPE-Neuro includes symptoms of intracranial pressure, epileptic seizures, sensory disturbances, sensitive deficits, motor disturbances, dysphagia, spasticity, vegetative disturbances, neuropsychological disorders, quantitative disturbance of consciousness, symptoms of delirium, change in personality, and loss of autonomy. All items have been discussed carefully among a multi-disciplinary team of neurologists and palliative care physicians (H.G., R.V., M.E., A.K.).

The response categories for both HOPE-SP-CL and HOPE-Neuro range from 0 (none) to 3 (severe). The total score is obtained by summing the item scores, that is, HOPE-SP-CL 0-51, HOPE-Neuro 0-39, and HOPE + 0-90. The ECOG performance status is used as standard criteria by clinicians to examine a patient's daily living activities and how a disease affects a patient's level of functioning. As such, we applied the ECOG as external validation criterion following Stiel et al. ${ }^{14}$ It has scores ranging from $0-5$, wherein 0 is fully active and 5 is dead. However, at baseline, the highest possible score given was 4 , completely disabled.

\section{"Surprise"-question}

The 12-SQ, in this study utilized as a second external validation criterion, is commonly used for prognosis estimation and to predict the need for integration of palliative care services into the care of patients..$^{29,30,34}$ It was answered by the attending neurologist based on clinical impression. Patients who were given a poor prognosis ("No, I would not be surprised") were then classified as "No"-group while patients who were given a good prognosis ("Yes, I would be surprised") were allocated to the "Yes"-group.

\section{Statistical analyses}

Descriptive statistics were calculated using SPSS software (v.25, SPSS, Inc., Chicago, IL). To test for normality, the Kolmogorov-Smirnov Test was applied.

A multitude of statistical tests was performed to assess the validity of $\mathrm{HOPE}+$. Construct validity was measured by a factor analysis and by evaluating discriminant validity. The exploratory factor analysis was conducted using the principal axis method with the direct oblimin rotation. This approach allows for creation of meaningful factors potentially correlating with each other. Only factors with an eigenvalue $>1$ were extracted. Prior to extraction of 
factors, we assessed the suitability of the data for factor analysis using the Kaiser-Meyer-Olkin Measure of Sampling Adequacy (KMO) and Bartlett's test of sphericity. As direct comparison to the direct oblimin approach (oblique rotation allowing factors to correlate), we also performed factor analysis using the more traditional varimax approach (orthogonal rotation keeping factors independent/uncorrelated). Discriminant validity was assessed by evaluating the dependency with the ECOG performance status. ${ }^{18,19,35}$ This should allow for a potential differentiation of patients with higher symptom burden who are impacted more severely in their level of functioning compared to patients with lower symptom burden and minimal impact on their daily living abilities. First, Spearman's rank correlation coefficients were computed to test the association between HOPE sum scores and ECOG. Second, pair-wise comparisons of HOPE sum scores stratified by ECOG were calculated using the Kruskall-Wallis $H$ Test for HOPE-SP-CL and HOPE + . Post hoc comparisons were carried out using Bonferroni's procedure at $p<0.05$. To extend the discriminatory power of both scales stratified by ECOG to the classification results of the 12-SQ, the "Yes"-group was statistically compared to the "No"-group. The combination of using the ECOG with the 12-SQ is plausible as lower functioning patients are more likely to die within the next 12 months than highly functioning patients. ${ }^{30}$ Third, the Mann-Whitney $U$ Test was used to test whether HOPE + could distinguish between patients who were expected to survive the allocated period of 1 year according to the physicians' response to the 12-SQ at time of admission and those with a poorer prognosis who might then be appropriate for referral into palliative care. Mean values and standard deviation $(S D)$ are reported for ease of understanding instead of median and quartiles.

Discriminant validity was extended after ascertaining the patients' survival status. Following the initial analysis, Spearman's rho was calculated between HOPE sum scores and survival status. The Mann-Whitney $U$ Test was then applied to differentiate deceased patients from patients who were still alive after 12 months, with and without stratification by ECOG.

To assess whether one of the two scales is diagnostically superior, the Mann-Whitney $U$ Test was first applied comparing weighted scores (by number of items) of both scales in the 12-SQ "yes" versus "no" group as well as in the survival status "deceased" versus "still alive" group of patients. Logistic regression analyses were then conducted for both scales to identify which scale best predicted 12-month mortality. The predictive validity of both scales was further evaluated comparing the areas under the curve (AUCS) by means of receiver operating characteristics (ROCs) analyses. ROC analyses allow for graphical plotting of sensitivity and specificity for all possible cut-off points. We chose for an optimal cut-off that maximizes both sensitivity and specificity, aiming for higher sensitivity relative to specificity. The AUCs are defined as measures of diagnostic accuracy.

\section{Results}

\section{Demographic information}

From 10 August 2016 until 10 March 2017, a total of 634 patients were newly admitted to the Dr. Becker Rhein-Sieg-Klinik in Nümbrecht, Department of Neurorehabilitation. Only patients-or their legal representatives-who gave written informed consent, being diagnosed with a "palliative care-relevant illness" and of whom complete data sets existed were evaluated. Therefore, 371 patients could not be recruited into the current study due to missing informed consent $(n=137)$, "non-palliative care-relevant illnesses" $(n=16)$, or incomplete data sets $(n=218)$. Demographical characteristics of the remaining 263 participants are presented in Table 1. Both the classification of diagnoses and rehabilitation phases are described in detail elsewhere. ${ }^{30}$ Each HOPE + assessment was complete and lasted approximately $15 \mathrm{~min}$.

At time of 12-month follow-up, 190 patients were still alive, 33 have deceased, and 40 were considered dropouts due to unattainability via the phone.

\section{Construct validity}

Factor analysis. Exploratory factor analysis revealed six factors, explaining $56.3 \%$ of the total variance. The suitability of the data for factor analysis was confirmed by the Bartlett's test $(p<0.001)$ and the KMO (0.890). Factor loading coefficients of $\geqslant 0.3$ on at least one factor were considered significant for the current study, ${ }^{36}$ factor loading coefficients of $\geqslant 0.2$ were considered acceptable if deemed clinically important. As a result, additional symptoms (0.194) were removed from further analyses. Factor loadings on epileptic seizures (0.201, factor 1), dyspnea (-0.293, factor 4), and wound care $(0.203$, factor 5$)$ were weak but were maintained due to their clinical importance. The results of the rotated loadings of the remaining 29 HOPE + items on each of the six factors are shown in Table 2 (the results of the varimax approach can be found in the Supplemental File S2 and are comparable, yet not identical to the results of the direct oblimin approach). Extracted factors showed moderate intercorrelations of $r=-0.543-0.525$.

Discriminant validity. Correlation analyses revealed moderate to strong positive correlations between ECOG and HOPE-SP-CL $(r s(261)=0.646, p<0.001)$ as well as HOPE + sum scores $(r s(261)=0.724, p<0.001)$. 
Table 1. Demographic data at baseline $(n=263)$.

\begin{tabular}{|c|c|c|}
\hline & $n$ & $\%$ \\
\hline \multicolumn{3}{|l|}{ Age (years) } \\
\hline Mean \pm standard deviation & $63 \pm 14$ & \\
\hline Range & $22-90$ & \\
\hline \multicolumn{3}{|l|}{ Sex } \\
\hline Male & 153 & 58.2 \\
\hline Female & 110 & 41.8 \\
\hline \multicolumn{3}{|l|}{ Primary diagnoses } \\
\hline Brain injury & 13 & 4.9 \\
\hline Critical illness polyneuropathy & 11 & 4.2 \\
\hline Dementia syndrome & 2 & 0.8 \\
\hline Hypoxic brain damage & 2 & 0.8 \\
\hline Infection of CNS & 21 & 8.0 \\
\hline Intracerebral hemorrhage & 24 & 9.1 \\
\hline Ischemic cerebral infarction & 131 & 49.8 \\
\hline Multiple sclerosis & 14 & 5.3 \\
\hline Neurodegenerative disorders & 29 & 11.0 \\
\hline Primary brain tumors & 9 & 3.4 \\
\hline Subarachnoid hemorrhage & 4 & 1.5 \\
\hline Subdural hematoma & 3 & 1.1 \\
\hline \multicolumn{3}{|l|}{ Secondary diagnoses } \\
\hline Bronchopulmonary diseases & 54 & 20.5 \\
\hline Cardiovascular diseases & 177 & 67.3 \\
\hline Diseases of the musculoskeletal system & 17 & 6.5 \\
\hline Infectious diseases & 19 & 7.2 \\
\hline $\begin{array}{l}\text { Malignancies (excluding primary brain } \\
\text { tumors) }\end{array}$ & 16 & 6.1 \\
\hline Neurological and psychiatric diseases & 36 & 13.7 \\
\hline Other internal diseases & 88 & 33.5 \\
\hline \multicolumn{3}{|l|}{ Eastern Cooperative Oncology Group } \\
\hline Grade 0: Fully active & 22 & 8.4 \\
\hline Grade 1: Restricted in physical activity & 120 & 45.6 \\
\hline $\begin{array}{l}\text { Grade 2: Ambulatory and capable of } \\
\text { self-care }\end{array}$ & 50 & 19.0 \\
\hline Grade 3: Capable of limited self-care & 56 & 21.3 \\
\hline Grade 4: Disabled & 15 & 5.7 \\
\hline \multicolumn{3}{|l|}{ Rehabilitation phase ${ }^{a}$} \\
\hline Phase B & 25 & 9.5 \\
\hline Phase C & 105 & 39.9 \\
\hline Phase D & 133 & 50.6 \\
\hline
\end{tabular}

CNS: central nervous system.

aGerman classification system characterizing type and intensity of neurological rehabilitation:

Phase B, early rehabilitation: Patients who are severely affected and might still suffer from disorders of consciousness. Intensive medical treatment may be required.

Phase C, subsequent rehabilitation: Patients still depend on high nursing and medical care. The aim is intensive mobilization.

Phase $\mathrm{D}$, medical rehabilitation: Patients can actively participate in rehabilitation measures. The aim is regaining independence in activities of daily life.

Pair-wise comparisons of HOPE + (Figure 1, top) and HOPE-SP-CL sum scores (Figure 1, bottom) stratified by ECOG showed significant differences in their level of functioning. Only patients with either high (grade 0 vs 1 ) or low (grade 3 vs 4) levels of functioning did not differ significantly for either HOPE scale. High discriminant validity was further confirmed by increasing sum scores with worsening ECOG for HOPE + (Table 3a, top) and HOPE-SP-CL (Table 3a, bottom), and additionally supported by adding the 12-SQ prognosis "yes" versus "no." These two groups can statistically be differentiated only within ECOG 4.

Sum scores of HOPE-SP-CL ("yes": $6.03 \pm 4.465$, "no": $13.87 \pm 9.629, \quad p<0.001)$ and HOPE + ("yes": $10.33 \pm 6.600$, "no": $24.77 \pm 17.549, p<0.001)$ differed significantly between the 12-SQ groups.

After ascertaining the patient's survival status, correlation analyses indicate low discriminatory power of survival for HOPE-SP-CL $(r s(221)=0.261, p<0.001)$ and HOPE $+(r s(221)=0.303, p<0.001)$. Similarly, no significant difference was found for survival status stratified by ECOG for neither HOPE + (Table 3b, top) nor HOPE-SP-CL (Table 3b, bottom). However, sum scores increased with ECOG for both groups (Table 3). Conversely, sum scores of HOPE-SP-CL (deceased: $12.27 \pm 8.893$, still alive: $6.44 \pm 4.879, \quad p<0.001$ ) and HOPE + (deceased: $22.24 \pm 15.879$, still alive: $11.05 \pm 7.310, p<0.001$ ) were significantly different for survival status irrespective of the level of functioning.

\section{Predictive validity}

Comparison of weighted HOPE scores resulted in null findings for both the 12-SQ and the survival groups (Table 4). Regression analyses revealed that HOPE-SP-CL and HOPE + added significantly to the model $(\beta=0.131$, odds ratio $(\mathrm{OR})=1.140,95 \%$ confidence interval $(\mathrm{Cl})=1.077-$ 1.206, $p<0.001, \quad$ and $\beta=0.093, \quad O R=1.098, \quad 95 \%$ $\mathrm{Cl}=1.056-1.141, \quad p<0.001$, respectively). Increasing scores of both scales were associated with an increased likelihood of dying. Operating characteristics of both scales are presented in Figure 2. The AUCs confirm that HOPE + and HOPE-SP-CL can discriminate fairly well between survival groups. Pairwise comparisons revealed a slight tendency toward superior diagnostic accuracy for the HOPE + relative to the HOPE-SP-CL; however, this difference was not significant $(0.746 \pm 0.049,95 \%$ $\mathrm{Cl}=0.650-0.842$ vs $0.712 \pm 0.049,95 \% \mathrm{Cl}=0.616-0.808$, respectively). When optimally chosen for a balanced trade-off between sensitivity and specificity, our results show identical sensitivity (HOPE + : 0.727 vs HOPE-SP-CL: 0.727 ) and comparable specificity (HOPE+: 0.621 vs HOPE-SP-CL: 0.584 ) at a cut-off of 11.5 for the HOPE + and 6.5 for the HOPE-SP-CL.

\section{Discussion}

The purpose of this study was to evaluate the validity of $\mathrm{HOPE}+$, a combination of HOPE-SP-CL ${ }^{14}$ and HOPE-Neuro, for use in neurological health care settings. Our results 
Table 2. Rotated factor loadings of the HOPE + .

\begin{tabular}{|c|c|c|c|c|c|c|}
\hline \multirow[t]{2}{*}{ Item } & \multicolumn{6}{|c|}{ Factors } \\
\hline & 1 & 2 & 3 & 4 & 5 & 6 \\
\hline \multicolumn{7}{|l|}{ Factor 1: Neuropsychiatric symptoms } \\
\hline Quantitative disturbance of consciousness & 0.936 & & & & & \\
\hline Symptoms of delirium & 0.880 & & & & & \\
\hline Dysphagia & 0.627 & & & & & \\
\hline Disorientation/confusion & 0.542 & & & & & \\
\hline Change in personality & 0.540 & & & & & \\
\hline Neuropsychological disorders & 0.471 & & & & & \\
\hline Vegetative disturbances & 0.414 & & & & & \\
\hline Sensory disturbances & 0.375 & & & & & \\
\hline Spasticity & 0.330 & & & & & \\
\hline Epileptic seizures & 0.201 & & & & & \\
\hline \multicolumn{7}{|l|}{ Factor 2: Intracranial pressure symptomatology } \\
\hline Nausea & & 0.977 & & & & \\
\hline Vomiting & & 0.753 & & & & \\
\hline Symptoms of intracranial pressure & & 0.436 & & & & \\
\hline \multicolumn{7}{|l|}{ Factor 3: Increasing need for care and assistance } \\
\hline Motor disturbances & & & 0.816 & & & \\
\hline Assistance with activity of daily living & & & 0.792 & & & \\
\hline Loss of autonomy & & & 0.607 & & & \\
\hline Overburdening of family & & & 0.439 & & & \\
\hline Organization of care & & & 0.437 & & & \\
\hline Sensation deficit & & & 0.434 & & & \\
\hline \multicolumn{7}{|c|}{ Factor 4: Psychological burden and strongly associated symptoms } \\
\hline Anxiety & & & & -0.915 & & \\
\hline Tension & & & & -0.878 & & \\
\hline Feeling depressed & & & & -0.747 & & \\
\hline Dyspnea & & & & -0.293 & & \\
\hline \multicolumn{7}{|l|}{ Factor 5: Additional physical symptoms } \\
\hline Pain & & & & & 0.922 & \\
\hline Constipation & & & & & 0.347 & \\
\hline Wound care & & & & & 0.203 & \\
\hline \multicolumn{7}{|l|}{ Factor 6: Powerlessness } \\
\hline Tiredness & & & & & & 0.650 \\
\hline Loss of appetite & & & & & & 0.577 \\
\hline Weakness & & & & & & 0.344 \\
\hline
\end{tabular}

HOPE + : extension of the symptom checklist of the core documentation system Hospice and Palliative Care Evaluation by neurological symptoms.

showed good construct validity, with six extracted factors and acceptable discriminatory power using the ECOG performance status. Our data further suggest that HOPE + is a suitable scale to capture palliative symptoms in neurological patients.

To the best of our knowledge, there is only one validated palliative care outcome measurement that incorporates neurological symptoms in patients with long-term neurological conditions, named IPOS Neuro-S8. ${ }^{36} \mathrm{~A}$ major difference between the IPOS Neuro-S8 and HOPE + is that the latter evaluates the incidence and intensity of symptom burden while the IPOS Neuro-S8 assesses the impact of symptoms on a patients' everyday life within the past 3 days. Also, the IPOS Neuro-S8 has not been validated in the German language and in Germany, HOPE has been established nationwide. HOPE + can now be translated into different languages and validated. It can then be of use in neurological health care settings around the world assessing the incidence and intensity of symptom burden which will help to identify neurological patients in need of palliative care.

\section{Construct validity}

The factor analysis generated a six-factor structure of multidimensional domains of symptoms. Factor 1 consists of 10 neuropsychiatric items, 9 from HOPE-Neuro and the 10th representing the neuropsychiatric category of HOPE-SP-CL, 


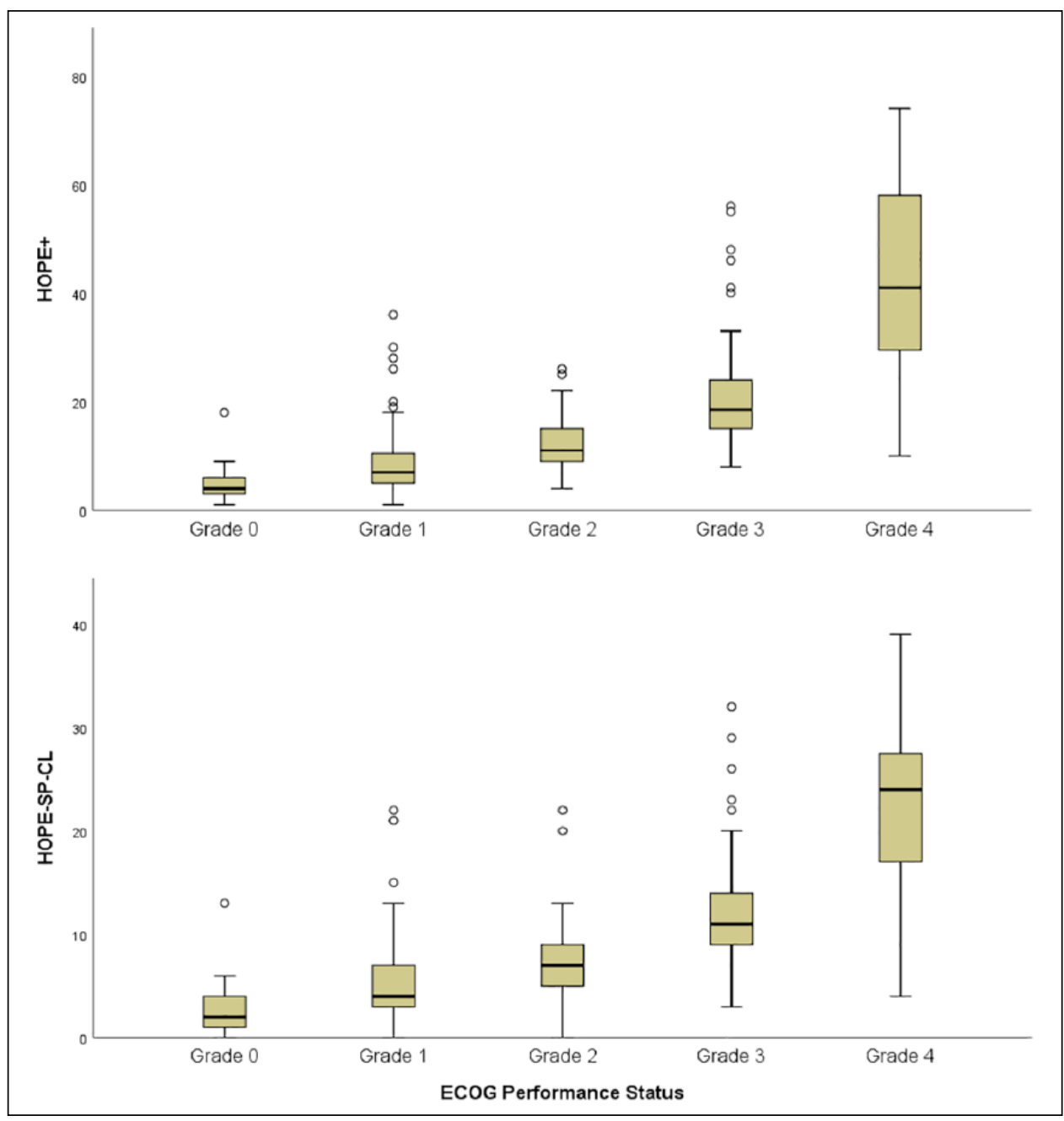

Figure 1. Pair-wise comparisons of the sum score of the HOPE + (top) and the HOPE-SP-CL (bottom) of patients stratified by ECOG performance status. All pair-wise comparisons were statistically different from each other at $p<0.05$ (Bonferroni corrected), except grade 0 versus 1 and grade 3 versus 4 .

Table 3. Mean sum scores of both the HOPE + (top) and the HOPE-SP-CL (bottom) by ECOG status performance and $12-\mathrm{SQ}^{\mathrm{a}}$ (a) and survival status (b).

\begin{tabular}{|c|c|c|c|c|c|c|c|}
\hline & a. & "No"-group & "Yes"-group & $p$ & b. Deceased & Still alive & $p$ \\
\hline \multirow[t]{5}{*}{ HOPE + } & ECOG 0 & $2.00(n=1)$ & $5.29 \pm 3.67(n=21)$ & 0.273 & $6.00(n=1)$ & $4.38 \pm 2.28(n=16)$ & 0.588 \\
\hline & ECOG 1 & $13.15 \pm 10.83(n=13)$ & $7.91 \pm 4.59(n=107)$ & 0.210 & $7.67 \pm 4.41(n=6)$ & $8.33 \pm 5.10(n=97)$ & 0.827 \\
\hline & ECOG 2 & $14.88 \pm 5.30(n=8)$ & $12.21 \pm 4.83(n=42)$ & 0.194 & $13.20 \pm 3.56(n=5)$ & $12.26 \pm 5.17(n=38)$ & 0.543 \\
\hline & ECOG 3 & $23.81 \pm 13.69(n=27)$ & $19.34 \pm 6.83(n=29)$ & 0.522 & $21.08 \pm 12.10(n=12)$ & $11.17 \pm 5.09(n=36)$ & 0.820 \\
\hline & ECOG 4 & $48.00 \pm 14.92(n=12)$ & $18.67 \pm 7.57(n=3)$ & $0.009^{b}$ & $40.33 \pm 13.23(n=9)$ & $24.33 \pm 15.63(n=3)$ & 0.145 \\
\hline \multirow{5}{*}{ HOPE-SP-CL } & ECOG 0 & $2.00(n=1)$ & $3.05 \pm 2.87(n=21)$ & 0.909 & $4.00(n=1)$ & $2.31 \pm 1.70(n=16)$ & 0.353 \\
\hline & ECOG 1 & $7.92 \pm 7.27(n=13)$ & $4.65 \pm 3.31(n=107)$ & 0.252 & $4.33 \pm 2.88(n=6)$ & $4.88 \pm 3.64(n=97)$ & 0.766 \\
\hline & ECOG 2 & $9.25 \pm 3.06(n=8)$ & $7.10 \pm 4.05(n=42)$ & 0.057 & $8.20 \pm 2.17(n=5)$ & $7.21 \pm 4.33(n=38)$ & 0.241 \\
\hline & ECOG 3 & $13.41 \pm 7.96(n=27)$ & $11.28 \pm 4.79(n=29)$ & 0.411 & $11.50 \pm 7.404(n=12)$ & $11.17 \pm 5.09(n=36)$ & 0.582 \\
\hline & ECOG 4 & $25.42 \pm 8.73(n=12)$ & $10.67 \pm 5.57(n=3)$ & $0.018^{b}$ & $21.78 \pm 8.043(n=9)$ & $12.67 \pm 8.08(n=3)$ & 0.064 \\
\hline
\end{tabular}

$\mathrm{HOPE}+$ : extension of the symptom checklist of the core documentation system Hospice and Palliative Care Evaluation by neurological symptoms; HOPE-SP-CL: symptom checklist of the core documentation system Hospice and Palliative Care Evaluation; ECOG: Eastern Cooperative Oncology Group. Numbers represent mean $\pm S D$ for ease of understanding instead of median and quartiles. Statistical analyses were done using non-parametric tests due to the skewed distribution of the data.

aPatients were assigned into two groups, depending on the neurologist's response to the 12-months "surprise" question (12-SQ). Those given a poor prognosis were categorized as the 12-SQ "No"-group while those who were given a good prognosis were classified as the 12-SQ "Yes"-group. ${ }^{\mathrm{b}}$ significant differences at $p<0.05$ (Bonferroni corrected). 
Table 4. Comparison of weighted HOPE scores for the 12-SQ (top) and survival groups (bottom).

\begin{tabular}{llll}
\hline & HOPE-SP-CL & HOPE + & $p$ \\
\hline "No"-group $(n=61)$ & $0.82 \pm 0.567$ & $0.83 \pm 0.585$ & 0.874 \\
"Yes"-group & $0.36 \pm 0.263$ & $0.34 \pm 0.220$ & 0.246 \\
( $n=202)$ & & & \\
Deceased $(n=33)$ & $0.72 \pm 0.523$ & $0.74 \pm 0.529$ & 0.555 \\
Still alive $(n=190)$ & $0.38 \pm 0.287$ & $0.37 \pm 0.244$ & 0.362 \\
\hline
\end{tabular}

HOPE+: extension of the symptom checklist of the core documentation system Hospice and Palliative Care Evaluation by neurological symptoms; HOPE-SP-CL: symptom checklist of the core documentation system Hospice and Palliative Care Evaluation.

Numbers represent mean $\pm S D$ for ease of understanding instead of median and quartiles. Statistical analyses were done using nonparametric tests due to the skewed distribution of the data.

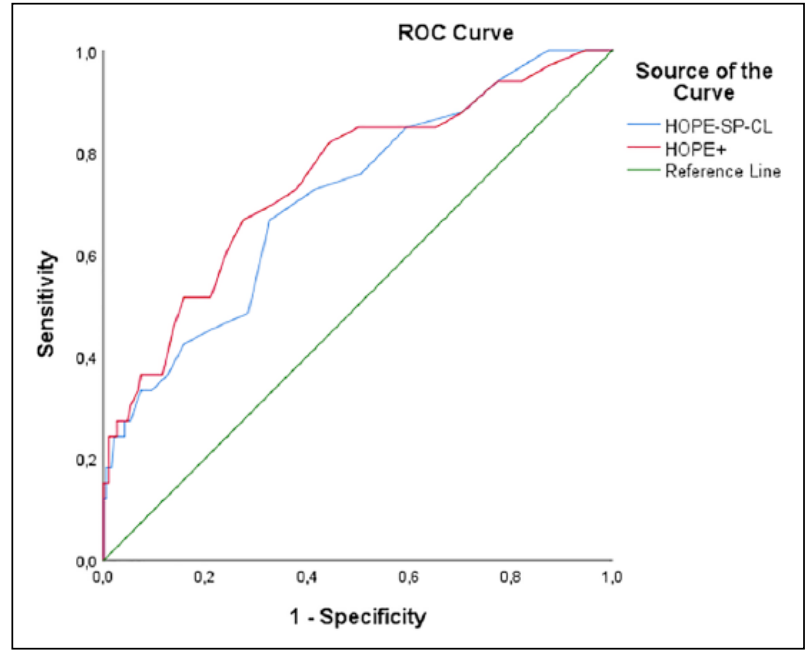

Figure 2. ROC curve showing the trade-off between sensitivity and specificity for the HOPE + and HOPE-SP-CL.

so we subsumed them under "neuropsychiatric symptoms." The second factor "intracranial pressure symptomatology" contains three items including nausea and vomiting which are not unique to intracranial pressure but are common symptoms thereof. The combined loading of both items onto one factor is in good agreement with the factor structure reported in previous studies.14,36 In fact, Stiel et al. ${ }^{14}$ performed an exploratory factor analysis on HOPE$\mathrm{SP}-\mathrm{CL}$ as part of the validation process and found comparable factor loadings to factors $2-6$ in the present study. Interestingly, three neurological items-motor disturbance, loss of autonomy, and sensation deficit-additionally loaded on factor 3 , corresponding to the "increasing need for care and assistance." The items epileptic seizures, dyspnea, and wound care loaded low on factors 1,4 , and 5 , respectively. Nevertheless, we maintained them in the analysis as they either relate well to their respective factors (dyspnea generally occurs together with anxiety and tension) and/or they are common symptoms in both neurological and oncological palliative care patients and, as such, convey clinically relevant information. ${ }^{37-40}$ The moderate intercorrelations of all six factors suggest a combination of both separable but also related dimensions. The latter indicates that the combination of HOPE-SP-CL and HOPE-Neuro can be used as one main construct for assessing symptom burden in neurological patients. We have thus succeeded in creating six meaningful factors correlating with each other to a satisfactory degree. This is in line with previous research suggesting the inclusion of additional symptoms when using the HOPE-SP-CL for specific patient groups such as neurological patients. ${ }^{14}$

Discriminant validity suggests satisfactory psychometric properties in differentiating highly functional patients with lower symptom burden from patients with lower performance status and higher symptom burden. Considerable differences between the 12-SQ groups in relation to their functional ability seem plausible as a physicians' prognosis estimation is based on, inter alia, clinical impression of the patients' functional abilities. ${ }^{41}$ We thus added the 12-SQ as external criterion to our analysis and found that patients in the "No"-group presented with higher symptom burden than patients in the "Yes"-group, ${ }^{30}$ both with and without stratification by performance status. In fact, discriminant validity in differentiating patients with respect to $12-\mathrm{SQ}$ was statistically confirmed for patients with the lowest performance status. In contrast, discriminatory power of survival status within 12 months stratified by ECOG was weak but improved with omission of ECOG. A potential explanation is the low statistical power arising from the lower sample size in the group of deceased patients together with the restricted number of severely ill patients. Our data show that the difference in symptom burden between survival groups increases with ECOG. This suggests that discriminatory power in identifying patients at risk of deceasing improves with the degree of functional disability, which seems reasonable and is in accordance with the results of a previous study showing that ECOG in combination with age and dysphagia is a significant predictor for prognosis estimation. ${ }^{30}$

\section{Predictive validity}

Our analyses suggest that HOPE + is a suitable instrument to detect neurological patients with high symptom burden who might be at risk of dying. To enhance the predictive power of survival in neurological patients, we propose the inclusion of the 12-SQ as the combination of the items age, dysphagia, and overburdening of family with the 12 -SQ have great predictive accuracy for 12 months survival and thus facilitate early referral into palliative care..$^{30}$

Although statistical comparison of the AUCs of both scales revealed no significant difference, the operating characteristics of the HOPE + were slightly higher compared to those of the HOPE-SP-CL indicating a tendency toward 
superiority in our sample of neurological patients. Similarly, we found slightly greater specificity for the HOPE+ compared to the HOPE-SP-CL, while the sensitivity of both scales remained identical. Our sample was recruited from one neurological rehabilitation clinic and the majority of patients were in rehabilitation phase $D$ already. Thus, we have reason to assume that the trend toward greater diagnostic accuracy might improve by including more patients from further neurological institutions treating patients with more advanced disease trajectories. Further studies are needed to verify the author's hypothesis.

\section{Clinical relevance}

HOPE + is a promising tool to evaluate the palliative symptom burden of neurological patients. From a clinical point of view, it facilitates the characterization of neurological patients in need of palliative care in the clinical routine of neurologists more so than using the HOPE-SP-CL alone. By using it, patients at risk of dying within the next 12 months could be identified, corroborated by adding the 12-SQ. The tool might aid to determine when the palliative care approach should be integrated into the care of this patient population. This is of particular importance as neurological patients are still underrepresented in palliative and hospice care structures. Reasons for this might be that (1) a sound validated outcome measurement addressing additional issues of this patient population is lacking in Germany and many other countries thus far (to the best of our knowledge, there is only the IPOS Neuro-S8), ${ }^{36}$ (2) disease trajectories of neurological patients are often longer and less predictable than those of oncological patients, $, 1,6$ and (3) health care professionals in neurology have shown restrained interest in palliative care, but this seems to be changing. ${ }^{1,4-7}$ Our approach addresses all these aforementioned aspects. HOPE + is feasible for health care professionals in demanding clinical neurological settings and can be included in the common anamnestic procedure.

\section{Conclusion}

Our results demonstrate satisfactory psychometric properties of $\mathrm{HOPE}+$, including a moderately correlated six-factor structure, acceptable discriminant validity, and promising diagnostic accuracy. We thus provide preliminary evidence for an extended validated outcome measure suitable to assess palliative care needs of neurological patients by a neurologist who is not trained in palliative medicine. This is essential as there is growing interest among neurologists to provide palliative care for their patients themselves or through referral into palliative and hospice care structures. HOPE + has potential to improve prognosis estimation and helps to identify the time to initiate a palliative care approach. Once established nationwide, it might also be appropriate for an international audience.

\section{Strengths and limitations}

Although our sample size is rather large compared to other validation studies, ${ }^{9,17,36,42}$ which could be considered a strength of our study, there are two major limitations to our study. The first one relates to the selection of our sample. All included patients were recruited from one neurological rehabilitation clinic, the majority was already in rehabilitation phase $D$ and suffered from an ischemic cerebral infarction. This limits the generalizability to other patient populations. Future studies are thus warranted investigating the diagnostic accuracy of HOPE + to corroborate our results, including a greater sample of patients in (1) neurological settings other than rehabilitation clinics, (2) clinically more severe rehabilitation phases, and (3) a wider range of neurological diagnoses.

Even though we were able to present a sufficient set of validation data using the two external validation criteria ECOG performance status scale and the 12-SQ, a second caveat of our study relates to the omission of additional instruments, preventing us from investigating a more comprehensive set of validity such as convergent validity as subtype of construct validity.

\section{Acknowledgements}

We thank all study participants and clinical personnel of the Dr. Becker Rhein-Sieg-Clinic, especially Britta Michler and Marion Birkholz for their valued assistance.

\section{Author Contributions}

M.E., H.G., R.V., A.K., and I.B. designed the study. M.E. recruited and assessed the majority of patients. K.D., I.B., and H.G. analyzed and interpreted the data. H.G. and K.D. drafted the manuscript; I.B., M.E., A.K., C.O., and R.V. revised it critically. All authors approved of the final version.

\section{Declaration of conflicting interests}

The author(s) declared no potential conflicts of interest with respect to the research, authorship, and/or publication of this article.

\section{Funding}

The authors received no financial support for the research, authorship, or publication of this article.

\section{ORCID iD}

Kim Dillen (iD https://orcid.org/0000-0002-0270-3338

\section{Supplemental Material}

Supplemental material for this article is available online. 


\section{References}

1. Turner-Stokes L, Sykes N, Silber E, et al. From diagnosis to death: exploring the interface between neurology, rehabilitation and palliative care in managing people with long-term neurological conditions. Clin Med 2007; 7(2): 129-136.

2. Oliver DJ, Borasio GD, Caraceni A, et al. A consensus review on the development of palliative care for patients with chronic and progressive neurological disease. Eur J Neurol 2016; 23(1): 30-38.

3. Rogers MD. National Landscape of Hospital-Based Palliative Care: Findings from the National Palliative Care Registry ${ }^{\mathrm{TM}}$, https://registry.capc.org/wp-content/uploads/2017/07 /National-Landscape-of-Hospital-Based-Palliative-Care _FINAL.pdf (2017, accessed 17 May 2019).

4. Maddocks I. Palliative care education for neurologists. In: Voltz R, Bernat JL, Borasio GD, et al. (eds) Palliative care in neurology (Contemporary Neurology Series), Vol. 69. New York: Oxford University Press, 2004, pp. 424-428.

5. Schuh LA, Biondo A, An A, et al. Neurology resident learning in an end-of-life/palliative care course. J Palliat Med 2007; 10(1): 178-181.

6. Boersma I, Miyasaki J, Kutner J, et al. Palliative care and neurology: time for a paradigm shift. Neurology 2014; 83(6): 561-567.

7. Hepgul N, Gao W, Evans CJ, et al. Integrating palliative care into neurology services: what do the professionals say? BMJ Support Palliat Care 2018; 8(1): 41-44.

8. Pace A, Dirven L, Koekkoek JAF, et al. European Association for Neuro-Oncology (EANO) guidelines for palliative care in adults with glioma. Lancet Oncol 2017; 18(6): e330-e340.

9. Hearn J and Higginson IJ. Development and validation of a core outcome measure for palliative care: the Palliative Care Outcome Scale. Qual Health Care 1999; 8(4): 219-227.

10. Bruera E, Kuehn N, Miller MJ, et al. The Edmonton Symptom Assessment System (ESAS): a simple method for the assessment of palliative care patients. J Palliat Care 1991; 7(2): 6-9.

11. Ellershaw JE, Peat SJ and Boys LC. Assessing the effectiveness of a hospital palliative care team. Palliat Med 1995; 9(2): 145-152.

12. Stolberg M. Die Geschichte der Palliativmedizin: medizinische Sterbebegleitung von 1500 bis heute. Frankfurt: Mabuse-Verlag, 2015.

13. Ostgathe $\mathrm{C}$, Gaertner J, Kotterba M, et al. Differential palliative care issues in patients with primary and secondary brain tumours. Support Care Cancer 2010; 18(9): 1157-1163.

14. Stiel S, Pollok A, Elsner F, et al. Validation of the symptom and problem checklist of the German Hospice and Palliative Care Evaluation (HOPE). J Pain Symptom Manage 2012; 43(3): 593-605.

15. Radbruch L, Nauck F, Ostgathe $C$, et al. What are the problems in palliative care? Results from a representative survey. Support Care Cancer 2003; 11(7): 442-451.

16. Radbruch L, Nauck F, Fuchs $M$, et al. What is palliative care in Germany? Results from a representative survey. J Pain Symptom Manage 2002; 23(6): 471-483.

17. Bausewein $C$, Fegg $M$, Radbruch $L$, et al. Validation and clinical application of the German version of the Palliative
Care Outcome Scale. J Pain Symptom Manage 2005; 30(1): 51-62.

18. Buccheri G, Ferrigno D, Tamburini M. Karnofsky and ECOG performance status scoring in lung cancer: a prospective, longitudinal study of 536 patients from a single institution. Eur J Cancer 1996; 32(7):1135-1141.

19. Conill C, Verger E, Salamero M. Performance status assessment in cancer patients. Cancer 1990; 65(8):1864-1866.

20. Golla H, Ale Ahmad M, Galushko M, et al. Glioblastoma multiforme from diagnosis to death: a prospective, hospital-based, cohort, pilot feasibility study of patient reported symptoms and needs. Support Care Cancer 2014; 22(12): 3341-3352.

21. Higginson IJ, Hart S, Silber E, et al. Symptom prevalence and severity in people severely affected by multiple sclerosis. $J$ Palliat Care 2006; 22(3): 158-165.

22. Oliver DJ, Campbell C, O'Brien T, et al. Medication in the last days of life for motor neuron disease/amyotrophic lateral sclerosis. Amyotroph Lateral Scler 2010; 11(6): 562-564.

23. Hensler $M$, Paul $S$, Abright $C$, et al. Progressive supranuclear palsy: living environment of the patients in Germany. Nervenarzt 2011; 82(2): 207-214.

24. Ostgathe C, Alt-Epping B, Golla H, et al. Non-cancer patients in specialized palliative care in Germany: what are the problems? Palliat Med 2011; 25(2): 148-152.

25. Golla H, Galushko M, Pfaff H, et al. Unmet needs of severely affected multiple sclerosis patients: the health professionals' view. Palliat Med 2012; 26(2): 139-151.

26. Lorenzl S, Nubling G, Perrar KM, et al. Palliative treatment of chronic neurologic disorders. Handb Clin Neurol 2013; 118: 133-139.

27. Grehl T. Diagnostics and therapy for amyotrophic lateral sclerosis. Fortschr Neurol Psychiatr 2013; 81(10): 592-603.

28. Lorenzl S. Multimorbidity of neurological patients in palliative care units. Nervenarzt 2014; 85(4): 409-416.

29. S3-Leitlinie Palliativmedizin für Patienten mit einer nicht heilbaren Krebserkrankun, https://www.awmf.org/uploads /tx_szleitlinien/128-0010LI_S3_Palliativmedizin_2015-07 .pdf (2015, accessed 14 September 2018).

30. Ebke $M$, Koch $A$, Dillen $K$, et al. The "surprise question" in neurorehabilitation-prognosis estimation by neurologist and palliative care physician: a longitudinal, prospective, observational study. Front Neurol 2018; 9: 792.

31. World Medical Association. World Medical Association Declaration of Helsinki: ethical principles for medical research involving human subjects. JAMA 2013; 310(20): 2191-2194.

32. Lindena G, Nauck F, Bausewein C, et al. Quality assurance in palliative medicine-results of the core documentation of 1999-2002. Z Arztl Fortbild Qualitatssich 2005; 99(9): 555-565.

33. Stiel S, Matthies DM, Seuss D, et al. Symptoms and problem clusters in cancer and non-cancer patients in specialized palliative care-is there a difference? J Pain Symptom Manage 2014; 48(1): 26-35.

34. White N, Kupeli N, Vickerstaff $\mathrm{V}$, et al. How accurate is the "surprise question" at identifying patients at the end of life? A systematic review and meta-analysis. BMC Med 2017; 15(1): 139. 
35. Oken MM, Creech RH, Tormey DC, et al. Toxicity and response criteria of the Eastern Cooperative Oncology Group. Am J Clin Oncol 1982; 5(6): 649-655.

36. Gao W, Crosby V, Wilcock A, et al. Psychometric properties of a generic, patient-centred palliative care outcome measure of symptom burden for people with progressive long term neurological conditions. PLOS ONE 2016; 11(10): e0165379.

37. Cabezón-Gutiérrez L, Khosravi-Shahi $P$, Custodio-Cabello $S$, et al. Opioids for management of episodic breathlessness or dyspnea in patients with advanced disease. Support Care Cancer 2016; 24(9): 4045-4055.

38. Fromantin I, Watson S, Baffie A, et al. A prospective, descriptive cohort study of malignant wound characteristics and wound care strategies in patients with breast cancer. Ostomy Wound Manage 2014; 60(6): 38-48.
39. Kazim SF, Shamim MS, Tahir MZ, et al. Management of penetrating brain injury. J Emerg Trauma Shock 2011; 4(3): 395-402.

40. Simon ST, Bausewein C, Schildmann E, et al. Episodic breathlessness in patients with advanced disease: a systematic review. J Pain Symptom Manage 2013; 45(3): 561-578.

41. Boussat $S$, El'rini T, Dubiez A, et al. Predictive factors of death in primary lung cancer patients on admission to the intensive care unit. Intensive Care Med 2000; 26(12): 18111816.

42. Stiel S, Matthes M, Bertram L, et al. Validierung der neuen Fassung des Minimalen Dokumentationssystems (MIDOS 2) für Patienten in der Palliativmedizin. Der Schmerz 2010; 24(6): 596-604. 